\title{
Physical conditions in $\mathbf{H}$ II galaxies
}

\author{
Carolina Kehrig and Eduardo Telles \\ Observatório Nacional, Rua General Jose Cristino 77, Sao Cristovao, \\ 20921-400 Rio de Janeiro, Brasil \\ François Cuisinier \\ Departamento de Astronomia, Universidade Federal do Rio de Janeiro, \\ 20080-090 Rio de Janeiro, Brasil
}

We present a spectrophotometric survey of $111 \mathrm{H}$ il galaxies. Observations were made at the ESO $1.52 \mathrm{~m}$ telescope, with the B\&C spectrograph. Our sample presents a great overlap with Terlevich et al. 1991. Additional targets come from the Calan-Tololo an Marseille catalogues. All objects have been observed and analysed homogeneously. We analyse their statistical properties and derive physical conditions (reddening, electron temperature, electron density), as well as chemical abundances.

In order to evaluate the quality of our survey, we compare our emission line intensities with Terlevich et al. (1991). The two data sets agree well within 0.1 dex. Furthermore, for low intensity lines, Terlevich et al. (1991) measurements are systematically higher than ours, which indicates a slightly higher $\mathrm{S} / \mathrm{N}$ in our spectra (Figure 1), as lines measured in low $\mathrm{s} / \mathrm{N}$ conditions tend to be systematically overestimated.

We were able to spatially resolve distinct regions of star formation in individual galaxies. We determine electron temperatures and abundances for each one of these regions separately. The variations of electron temperatures and oxygen abundances of the individual regions, compared with the integrated galaxy, are small and within observational uncertainties ( 0.1 dex for electron temperatures and 0.2 dex for oxygen abundances, see Figure 2). We also analysed fluctuations in extinction and electron densities, and found them to be sometimes important, especially for the extinction.

Thus, despite their 'blobbiness' and the significative variations in some physical properties, H II galaxies are chemicaly fairly homogeneous and well mixed. 


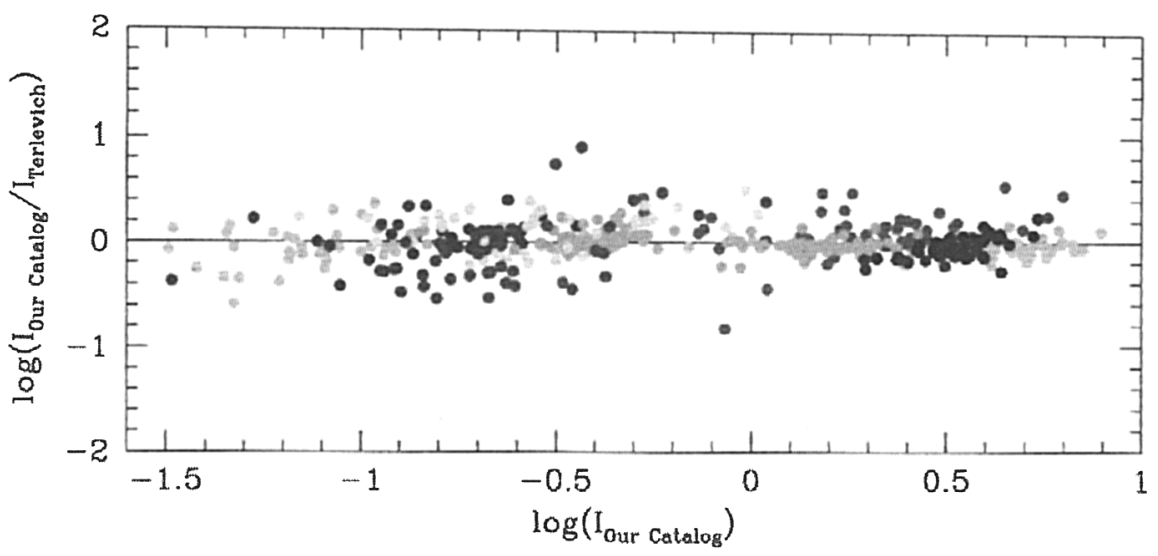

Figure 1. Comparison of our emission line intensities with Terlevich et al. (1991).
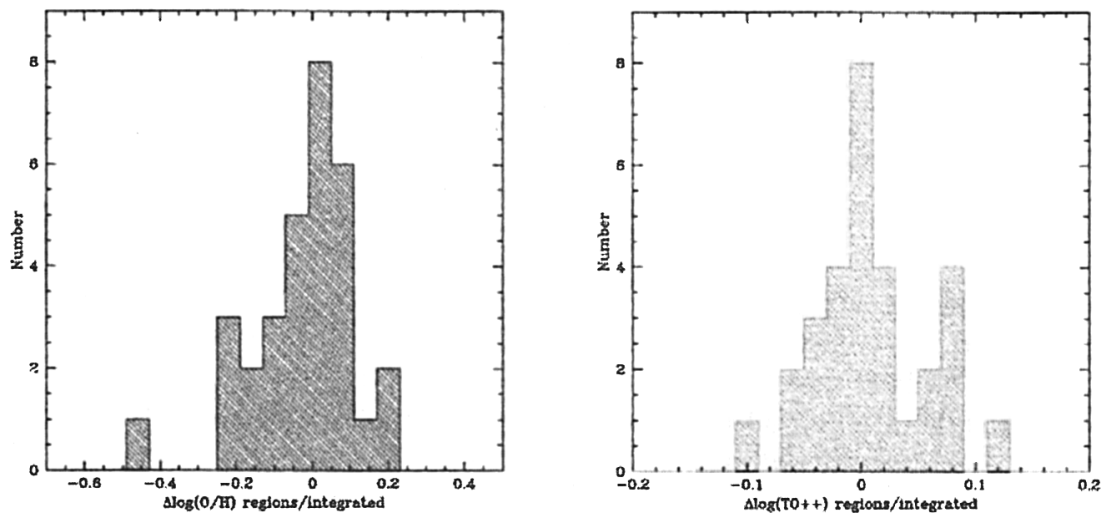

Figure 2. Electron temperatures and oxygen abundances of individual regions, compared to the integrated galaxies.

\section{References}

Maza, J., Ruiz, M.T., Gonzalez, L.E., Wischnjewsky, M., Peña, M. 1991, A\&AS 89, 389 (Calán-Tololo Survey III - H II Galaxies)

Surace, C., Conte, G. 1998, A\&AS 133, 171 (Marseille Schmidt Survey)

Terlevich, R., Melnick, J., Masegosa, J., Moles, M., Copetti, M.V.F. 1991, A\&AS 91, 285 